\title{
The new threats of Gram positive pathogens: re-emergence of things past
}

\author{
R A Howe, N M Brown, R C Spencer
}

...re-emergence of things past,

I sigh the lack of many a thing I sought,

And with old woes new wail my dear times' waste.

Sonnet No.30 (W Shakespeare, adapted)

\section{Introduction}

In recent years Gram positive bacteria have re-emerged as important pathogens, both in the community and hospital. To compound the problems, antimicrobial resistance, long considered the domain of Gram negative bacteria, is being increasingly exhibited by Gram positive strains.

This short review will look at renewed interest in previously uncommon pathogens "our old friends"-Corynebacterium diphtheriae and Streptococcus pyogenes; and the serious problems we face in treating patients infected with Staphylococcus aureus, Streptococcus pneumoniae and the enterococci.

\section{Streptococcus pyogenes}

Before the discovery of antibiotics, serious infections caused by $S$ pyogenes were common and were responsible for as many as $50 \%$ of post-partum deaths and the major cause of deaths in patients with burns before World War II. ${ }^{1}$ The infectious sequelae, such as rheumatic fever, were also common. The introduction of penicillin resulted in the organism being consigned to the history books. But not for long.

Since the mid-1980s an increased incidence of invasive disease has been reported from many parts of the industrialised world, including the USA, the UK and Scandinavia. ${ }^{2-4}$ Coincidentally, focal resurgence of rheumatic fever was reported in different areas in the USA, beginning in the Rocky Mountain area. ${ }^{5}{ }^{6}$ The predominant serotypes associated with rheumatic fever were $M 1,3$ and 18, which produce characteristic mucoid colonies. Changes in the prevalence of predominant serotypes continue to occur, which may cause the observed fluctuation in the incidence of streptococcal disease. In the UK, types 1,28 and 4 now predominate, ${ }^{7}$ whilst in the USA types 1,3 and 8 are prevalent. ${ }^{2}$ These new $M$ types are more invasive, cause increased mortality and are more likely to occur in clusters. This increased invasiveness may be related to the low level of immunity in populations where these $M$ types are less common. There is also evidence that patients with serious disease are infected by a unique clone. ${ }^{8}$ Of those studied, $90 \%$ had a characteristic restriction fragment length profile and were positive for the streptococcal pyogenic exotoxin (SPE) A gene.

Until recently, streptococcal isolates from cases of scarlet fever were only positive for SPE-B or SPE-C. ${ }^{9}$ Strains which produce SPE-A are now being isolated from patients with widespread cellulitis or necrotising fasciitis, associated with severe systemic toxicity, resembling staphylococcal toxic shock syndrome. ${ }^{10}$ Strains isolated before 1940 were also positive for SPE-A; so it seems that the more toxic form of scarlet fever was prevalent prior to the introduction of antibiotics, and is now re-establishing itself.

The term streptococcal toxic shock-like syndrome (TSLS) has been used to describe patients with hypotension and multisystem failure typical of the staphylococcal disease. ${ }^{11}$ This phenomenon can be partly explained by the fact that SPE-A shows $50 \%$ amino acid homology with staphylococcal enterotoxin B, one of the main toxins responsible for staphylococcal toxic shock syndrome. ${ }^{12}$ The clinical outcome of TSLS depends on the interaction between the microbial virulence factors ( $M$ protein and SPEs) and the immune status of the host. ${ }^{4}$

Penicillin remains the treatment of choice for streptococcal infection, but in patients with severe disease large numbers of the organisms can be found, and the efficacy of penicillin is thereby reduced. Two explanations have been proposed; the slower growth rate of streptococci when present in large numbers ${ }^{13}$ or the decreased expression of penicillin binding proteins (PBPs) in the presence of large inocula. ${ }^{14}$ Other antibiotics which have been found to be more effective in experimental animal models include clindamycin and erythromycin. ${ }^{15}$ The possibility of a future multivalent group A streptococcal vaccine has recently received a boost, following reports that a purified tetravalent $M$ protein produced significant antibody levels against all four serotypes of native $M$ proteins in a rabbit model. ${ }^{16}$

\section{Staphylococcus aureus}

$S$ aureus has been well known as a major pathogen for many years. It causes septicaemia, endocarditis, osteomyelitis, abscesses, pneumonia, wound infections, impetigo, boils, and a variety of toxin mediated diseases. In the pre-antibiotic era $S$ aureus bacteraemia carried a mortality of approximately $82 \%$ and despite the availability of effective antibiotic treatment, mortality remains high at $25-63 \%$. In recent years $S$ aureus has been newly implicated in further diseases such as toxic shock syndrome ${ }^{17}$ and Kawasaki syndrome. ${ }^{18}$ However, it is in the field of hospital infection control that $S$ aureus is causing increasing concern due to the emer- 
gence of increasingly resistant strains with high epidemic potential and virulence.

$S$ aureus was identified as a cause of hospital acquired wound infection in the $1880 \mathrm{~s}$. As streptococcal wound infections decreased in incidence $S$ aureus became the commonest cause of nosocomial wound infection in the 1940s. In 1980 the first national survey of infections in hospital identified $S$ aureus as responsible for $18 \%$ of all nosocomial infections and $33 \%$ of postoperative wound infections in the UK. ${ }^{19}$

$S$ aureus has displayed a great ability to develop resistance to a wide range of antibiotics. Soon after the introduction of penicillin the first resistant strain producing $\beta$-lactamase was reported $^{20}$ and penicillin resistance rapidly spread. In the early 1960 s $\beta$-lactamase stable penicillins such as flucloxacillin were developed which have become the mainstay of antibiotic treatment for $S$ aureus in the UK. In the laboratory flucloxacillin sensitivity of $S$ aureus is inferred from the sensitivity to the related compound methicillin. Resistance to methicillin is mediated by alterations to the PBPs on the cell surface which confers cross-resistance to all $\beta$-lactams including cephalosporins.

Strains of $S$ aureus resistant to methicillin (MRSA) were reported as early as $1961^{21}$ and were involved in nosocomial infection by $1966 .{ }^{22}$ During the 1970 s little notice was taken of MRSA - most strains were not multiply resistant to other classes of antibiotics and outbreaks of infection seemed to be manageable. During this time there was some suggestion that MRSA was inherently less virulent than methicillin sensitive $S$ aureus (MSSA). However, not surprisingly, it transpires that strains of MRSA vary in their virulence and epidemic potential just as MSSA.

Interest in $S$ aureus was re-kindled in the early 1980s with the recognition that a single strain was causing nosocomial outbreaks of infection in a number of hospitals in the southeast of England. A survey in 1988 identified 14 strains of MRSA which were affecting more than one hospital in England and Wales. ${ }^{23}$ These strains were termed epidemic MRSA (EMRSA) strains; EMRSA-1 (the SE England strain) was by far the most common, affecting 50 hospitals.

With the recognition of uncontrolled spread of EMRSA, infection control guidelines were published and have been updated. ${ }^{24}$ However, these have not been successful in controlling the problem. This is partly due to the nature of modern health provision with increased interhospital referral, increased reliance on agency nursing staff who may transfer between many hospitals, increased numbers of severely debilitated inpatients, and partly due to new emergent strains of MRSA with high epidemic potential. Since 1990 two new epidemic strains have emerged, EMRSA-15 and EMRSA-16. By June 1995, these strains were each affecting more than 80 hospitals. ${ }^{25}$ Of particular concern is EMRSA-16 which is resistant to many antistaphylococcal antibiotics including erythromycin, gentamicin, neomycin, ciprofloxacin, and trimethoprim. EMRSA-16 is generally sensitive to mupirocin but some isolates exhibit resistance. Mupirocin resistance is a problem as nasal mupirocin is the most effective and recommended treatment of MRSA carriers. ${ }^{24}$

At present, the mainstay of treatment for infections caused by MRSA is vancomycin. This antibiotic has been used for more than three decades without the development of resistance in $S$ aureus. However, there is concern that vancomycin resistance may emerge. The related glycopeptide teicoplanin is often used instead of vancomycin as it has a better side effect profile. Worryingly, there have been reports of the failure of teicoplanin treatment for $S$ aureus infection due to the emergence of teicoplanin resistance. ${ }^{26}$ Resistance to vancomycin has been reported in coagulase negative staphylococci ${ }^{27}$ and in enterococci (discussed later). Vancomycin resistance is still difficult to select for in vitro but transfer of resistance genes from vancomycin resistant enterococci (VRE) to $S$ aureus has already been achieved in the laboratory. ${ }^{28} \mathrm{With}$ the increasing use of vancomycin for treating MRSA infections and the spread of VRE in the same hospital population it would seem likely that there will be transfer of resistance between the genera. When this occurs in the patient population we shall return to the pre-antibiotic era for $S$ aureus.

\section{Enterococci}

Enterococci are found as normal gastrointestinal and vaginal flora, and are some of the most frequently isolated organisms from clinical specimens. In general, they are considered to be of low pathogenicity, and are much less frequently found as a cause of infection. In adults enterococci are particularly associated with infection of the urinary tract. They are also a well recognised cause of endocarditis, and bacteraemia may result from instrumentation of the urinary or gastrointestinal tract, or from infection of indwelling vascular lines.

There is evidence that enterococcal infections are becoming more common, particularly in immunocompromised patients, and they are now a major cause of nosocomial infection. ${ }^{29}$ This is predominantly infection related to invasive procedures, although selection pressure in favour of enterococci through antibiotic use, particularly cephalosporins, is important. The relative antibiotic resistance of enterococci makes treatment difficult, but the ability of enterococci to acquire new resistance is also a major cause for concern.

Enterococci are intrinsically less sensitive to $\beta$-lactam antibiotics than streptococci because they possess low affinity PBPs, and are generally regarded as being resistant to cephalosporins. ${ }^{30}$ However, a combination of penicillin or ampicillin and aminoglycoside is synergistic and has become the treatment of choice for serious enterococcal infection. $E$ faecium seems to be more resistant than $E$ faecalis; higher minimum inhibitory concentrations (MIC) to penicillin are seen (typically 8-32 $v$ 1-2 $\mathrm{mg} / \mathrm{l}$ ), and recently $E$ faecium showing high level resistance to gentamicin (MIC >2000 
$\mathrm{mg} / \mathrm{l}$ ) have become common, accounting for $>50 \%$ of isolates in some centres. These strains do not show synergy between $\beta$-lactam antibiotics and aminoglycosides. High level resistance to $\beta$-lactam antibiotics has also emerged (penicillin MIC $>200 \mathrm{mg} / \mathrm{l}$ ). This may be because of the production of $\beta$-lactamase (particularly in $E$ faecium, where $\beta$-lactamase production and high level resistance to aminoglycosides are commonly seen together), or (in $E$ faecium and $E$ raffinosus) result from overproduction of a low affinity PBP (PBP-5). ${ }^{30} 31$ Acquired resistance to chloramphenicol, macrolides, cotrimoxazole, and tetracycline may also occur. ${ }^{30}$

It is therefore understandable that there was considerable concern when initial reports of VRE appeared in $1988 .{ }^{32}{ }^{33}$ Since then, isolation of VRE has become more common and geographically widespread. VRE accounted for $14 \%$ of enterococci in intensive care units in the USA in $1993 .^{29}$ Many VRE, particularly isolates of $E$ faecium, also show high level resistance to $\beta$-lactam antibiotics and aminoglycosides.

Based on phenotypic characteristics, three main groups of resistance have been described: VanA, VanB and VanC. ${ }^{34}$ The VanA phenotype is inducible high level resistance to both vancomycin and teicoplanin. Resistance is readily transferable and is conferred by a plasmid gene designated van $A$, encoding an altered peptide in peptidoglycan synthesis which prevents binding of glycopeptides to the cell wall. ${ }^{34}$ VanB resistance is also inducible and transferable, but the exact site of the vanB gene is unknown. It confers moderate resistance to vancomycin, but not teicoplanin. Like VanA resistance, it is most commonly seen in $E$ faecium, but also occurs in $E$ faecalis. VanA resistance may also occur in $E$ avium. VanC resistance is constitutively expressed and the vanC gene is likely to be chromosomal. It is seen in $E$ gallinarum and $E$ casseliflavus and confers low level resistance to vancomycin alone.

The epidemiology of VRE is not completely understood, but the apparent rapid spread of these organsims is of great concern. The recent emergence of VRE not only jeopardises treatment of enterococcal infection, but threatens the potential spread of resistance to other organisms, particularly staphylococci. Risk factors for infection with VRE include inpatients of large hospitals ( $>500$ beds), length of hospital stay ( $>2$ weeks), severe underlying disease, haematological malignancy, and recent treatment with antibiotics, including vancomycin..$^{29} 3536$ Nosocomial transmission of VRE within hospitals is well documented. ${ }^{37}{ }^{38}$

Infection control measures to limit the spread of VRE have been published recently. ${ }^{39} 40$ These include increased surveillance for vancomycin resistance, measures to limit the unnecessary use of glycopeptides, and control measures to prevent spread within the hospital environment. It has been suggested that "because of the grave clinical problems posed by VRE, herculean measures (to prevent spread) seem eminently appropriate". ${ }^{39}$

\section{Pneumococci}

Streptococcus pneumoniae has been known as a major pathogen for over 100 years. Despite effective antimicrobial chemotherapy it continues to cause considerable morbidity and mortality: $S$ pneumoniae causes approximately $25 \%$ of community acquired pneumonia, of which if severe enough to require admission to an intensive therapy unit, the causative organism is most likely to be the pneumococcus. ${ }^{41}$ $S$ pneumoniae accounted for $10 \%$ of isolates from blood cultures and $18 \%$ of isolates from cerebrospinal fluid reported to the PHLS Communicable Disease Surveillance Centre (CDSC) in 1982-92 ${ }^{42} ; S$ pneumoniae causes up to $50 \%$ of episodes of otitis media and is a major cause of bacterial sinusitis and bronchitis.

Recently, there has been increasing concern over pneumococcal disease because of the increasing spread of penicillin resistant pneumococci. A pneumococcus is defined as intermediately resistant to penicillin if the strain has a MIC $>0.1 \mathrm{mg} / 1$ but $<1.0 \mathrm{mg} / 1$ and fully resistant if the MIC is $>2.0 \mathrm{mg} / \mathrm{l}$. Penicillin resistance was first selected in laboratory strains of pneumococcus soon after the release of penicillin in 1941. However, it was not until 1967 that a clinically significant pneumococcus with reduced penicillin sensitivity (MIC $0.6 \mathrm{mg} / \mathrm{l}$ ) was reported in an immunodeficient patient with pneumonia in Australia. In 1978 multiply resistant pneumococci with high level penicillin resistance were reported in South Africa ${ }^{43}$ In the late 1970 s penicillin resistant pneumococci were most prevalent in New Guinea, Israel, Poland, Spain, and South Africa, but they are now found in increasing numbers worldwide. ${ }^{44}$ For example, $41 \%$ of isolates from children in Atlanta, USA, in 1994 were penicillin resistant ${ }^{45}$; penicillin resistance in France rose from less than $1.1 \%$ in 1984 to $12 \%$ by $1990^{46}$; the incidence of penicillin resistant pneumococci in children in a university hospital in Korea in 1991-3 was $82 \% .{ }^{47}$ By comparison, only $2.5 \%$ of reported isolates from bacteraemia or meningitis reported to the PHLS CDSC in 1993 were resistant to penicillin. ${ }^{48}$

Resistance to penicillin in pneumococci is achieved by alteration of the high molecular weight PBPs. ${ }^{49}$ Genetic studies suggest that penicillin resistance in pneumococci has arisen through natural transformation and genetic recombination with other penicillin resistant species such as Streptococcus mitis, Streptococcus sanguis or Streptococcus mitior. There is a great deal of diversity among strains of pneumococci resistant to penicillin, but superimposed on this diversity there seem to be some clones with increased epidemic potential. For example, it seems that a clone of serotype $23 \mathrm{~F}$ displaying multiple drug resistance has spread from Spain and South Africa to the USA. ${ }^{50}$

The impact of penicillin resistance on the severity of disease has yet to be fully established. The main risk factors for infection by penicillin resistant pneumococci are similar to the risk factors for severe pneumococcal disease-extremes of age, hospitalisation, and 
previous antibiotic treatment..$^{51}$ As initial empiric treatment is likely to be ineffective it is not surprising that the outcome in some patients is poor. However, a study from Barcelona has suggested that the mortality due to pneumococcal pneumonia is not affected by the penicillin susceptibility of the pneumococcus. ${ }^{52}$

Treatment of infections caused by penicillin resistant pneumococci has been reviewed elsewhere. ${ }^{53}$ With intravenous treatment the concentrations of penicillin in the serum that can be achieved will greatly exceed the MIC of intermediately resistant and many fully resistant strains. Thus, a $\beta$-lactam remains the empiric drug of choice in this situation. However, if a highly resistant strain is suspected, vancomycin or a carbapenem should be considered. In the case of meningitis, penicillin is unlikely to be effective and the empiric therapy would be a third generation cephalosporin such as cefotaxime. Unfortunately, there have been reports of failure of cefotaxime treatment in meningitis due to penicillin resistant pneumococci that are also resistant to third generation cephalosporins. ${ }^{54}$ In this situation vancomycin or rifampicin, or both, has been suggested. Recent results show that such resistance to third generation cephalosporins is rare in the UK, with a prevalence of $<0.2 \% .^{55}$

As noted earlier, some clones of pneumococci seem to have increased epidemic potential and it is likely that the prevalence of penicillin resistant pneumococci in the UK will increase. If treatment is less effective there should be increased impetus for the development of a conjugate vaccine and a reassessment of the indications for vaccination and of our empiric antimicrobial treatment.

\section{Diphtheria}

Classic pharyngeal diphtheria results from infection with exotoxin producing strains of Corynebacterium diphtheriae. The importance of diphtheria lies, firstly, in the mortality of $5-10 \%$ of those infected (which has not changed greatly over the past 50 years), and, secondly, in the potential for person to person spread of the organism. In the UK diphtheria is now uncommon. Notification in children under the age of four years in London fell from approximately 100 cases per 10000 children in 1935 to one case per 10000 in $1950 .^{56}$ This followed introduction of diphtheria toxoid and increasing uptake of vaccination from $5 \%$ in 1935 to $77 \%$ in 1950 . Improved socioeconomic conditions have been important also. Vaccine uptake in the UK is currently $94 \% .{ }^{57}$ Between 1986 and 1993 only 33 toxigenic $C$ diphtheriae isolates were reported in England and Wales, the majority of which were imported from the Indian Subcontinent. ${ }^{58}$

There have been several recent changes, however, which are a cause for concern, and emphasise the importance of this traditional infectious disease. Since 1991 the incidence of pharyngeal diphtheria in countries of the former Soviet Union has increased dramatically. WHO figures show that there has been an increase in reported cases from 3100 in 1991 to 46000 in 1994 (table 1). ${ }^{59}$ Several factors
Table 1 Reported cases of diphtheria in countries of the former Soviet Union (figures from the WHO)

\begin{tabular}{ll}
\hline Year & No. of cases reported \\
\hline 1991 & 3100 \\
1992 & 5700 \\
1993 & 19500 \\
1994 & 46000 \\
\hline
\end{tabular}

have contributed to this epidemic, related to the social and economic changes occurring in the former Soviet Union at the time. For a variety of reasons, diphtheria immunisation rates fell in the 1980 s, so that by 1990 only $68 \%$ of Russian children had been vaccinated against diphtheria. ${ }^{59}$ Also, immunity in previously vaccinated adults may have fallen to subprotective levels, and natural immunity was poor because of the low incidence of diphtheria at the time. Therefore, the population was susceptible to infection when diphtheria was introduced by movement of large numbers of people following political changes in the early 1990s. The epidemic continued because of shortages of diphtheria vaccine and difficulties in improving vaccine uptake.

This increasing incidence of diphtheria is of concern to other countries. While most imported cases originate in either the Indian Subcontinent or Africa, diphtheria from the former Soviet Union has occurred in some European countries, but not, as yet, in the UK. It is known that some developed countries have low vaccine coverage. Also, protective antibody levels are not present in a significant proportion of previously vaccinated adults, particularly women, and therefore there is potential for further epidemic spread to occur if diphtheria is reintroduced. ${ }^{60}{ }^{61}$ The WHO Expanded Program on Immunisation in the European Region has stressed the importance of vaccination, and has set a target of $95 \%$ coverage in every country by $1995 . .^{62}$ In addition it has been suggested that a booster dose of diphtheria vaccine should be given to schoolleavers. ${ }^{63}$ The importance of effective diphtheria surveillance and immediate control measures has also been emphasised.

There is also concern about disease caused by non-toxigenic $C$ diphtheriae. The current status of $C$ diphtheriae carriage in the $\mathrm{UK}$ is unknown. As clinical diphtheria is now so uncommon many microbiology laboratories no longer routinely look for the organism in throat swabs, and it is very possible that its isolation from other sites would be overlooked. Despite this, isolation of non-toxigenic strains of $C$ diphtheriae has been more frequently reported. ${ }^{64}$ Increased pharyngeal carriage has been documented in homosexual men attending a genito-urinary clinic in London ${ }^{65}$ and in intravenous drug users. ${ }^{66}$ Invasive infection due to non-toxigenic $C$ diphtheriae is well described, and typing has shown that clusters of infection have often been caused by single strains. ${ }^{6768}$ This would be a particular cause for concern if these strains were able to become toxin producers. Lysogenic conversion to toxin production is possible in vitro, ${ }^{69}$ but its clinical significance is unknown. 


\section{Conclusions}

The resurgence of these Gram positive organisms is unquestionable and resistance to multiple antimicrobial agents continues apace. We need to be aware of these threats and hope that suitable alternatives to the classic or standard therapeutic agents are forthcoming.

1 Spencer RC. Invasive streptococci. Eur 7 Clin Microbiol Infect Dis 1995;14(Suppl 1):26-32.

2 Schwartz B, Fackham RR, Breiman RD. Changing epidemiology of group A streptococcal infections in the USA Lancet 1990;336:1167-71.

3 Strobeck S, Jepsen OB, Zimakoff J, Ronne T. Increased number of sporadic nosocomial group A streptococcal bacteraemia during a community epidemic. $¥$ Hosp Infect 1991;19:129-36.

4 Steven DL. Invasive Group A streptococcal infections. Clin Infect Dis 1992;14:2-13.

5 Bisno AL. Group A streptococcal infections and acute rheumatic fever. N Engl f Med 1991;325:783-93.

6 Denny FW Jr. A 45-year perspective on the streptococcus and rheumatic fever: the Edward $\mathrm{H}$ Kass lecture in infectious disease history. Clin Infect Dis 1994;19:1110-22.

7 Gaworzewska E, Colman G. Changes in the pattern of infection caused by Streptococcus pyogenes. Epidemiol Infect 1988;100:257-69.

8 Cleary PP, Kaplan EL, Handley JP, Wlazlo A, Kim NH, Hauser AR, et al. Clonal basis for resurgence of serious Streptococcus pyogenes disease in the 1980's. Lance 1992;339:518-21.

9 Hallas G. The production of pyrogenic exotoxins by Group A streptococci. $\mathcal{F}$ Hygiene 1985;95:47-57.

10 Talkington DF, Schwartz B, Black CM, Todd JK, Elliot J, Breiman RF, et al. Association of phenotypic and genotypic characteristics of invasive Streptococcus pyogenotypic characteristics of invasive Streptococcus pyotoxic shock syndrome. Infect Immun 1993;61:3369-74.

11 Forni AL, Kaplan EL, Schlivert PM, Roberts RB. Clinical and microbiological characteristics of severe group $A$ streptococcus infections and streptococcal toxic shock syndrome. Clin Infect Dis 1995;21:333-40.

12 Cone LA, Woodard DR, Schlievert PM, Tomory GS. Clinical and bacteriological observations of a toxic shock-like syndrome due to Streptococcus pyogenes. $N$ Engl $7 \mathrm{Med}$ 1987;317:146-9.

13 Stevens DL, Gibbons AE, Bergstroum R, Winn V. The Eagle effect revisited: efficacy of clindamycin, erythromycin and penicillin in the treatment of streptococcal myositis. $f$ Infect Dis 1988;158:23-8.

14 Stevens DL, Yan S, Bryant AE. Penicillin-binding protein expression at different growth stages determines penicillin efficacy in-vitro and in-vivo: an explanation for the inoculum effect. $\mathcal{F}$ Infect Dis 1993;167:1401-5.

15 Gemmell CG, Peterson PK, Schmeling D, Kim Y, Mathews $\mathrm{J}$, Wannamaker $\mathrm{L}$, et al. Potentiation of opsonisation and phagocytosis of Streptococcus pyogenes following growth in the presence of clindamycin. $f$ Clin Invest 1981; in the presenc.

16 Dale JB, Chiang EY, Lederer JW. Recombinant tetravalent group A streptococcal M protein vaccine. F Immunol 1993; 151:2188-94.

17 Shands KN, Schmid GP, Dan BB, Blum D, Guidotti RJ, Hargrett NT, et al. Toxic-shock syndrome in menstruating women: associated with tampon use and Staphylococcus women: associated with tampon use and Staphylococcus 1980;303: 1436-42.

18 Leung DYM, Meissner HC, Fulton DR, Murray DL, Kotzin BL, Schlievert PM, et al. Toxic shock syndrome toxin-secreting Staphylococcus aureus in Kawasaki syndrome. Lancet 1993;342:1385-8.

19 Meers PD, Ayliffe GAJ, Emmerson AM, Leigh DA, MayonWhite RT, Macintosh CA, et al. Report on the National Survey of Infections in Hospitals 1980. F Hosp Infect 1981; S2:1-11.

20 Spink WW, Ferris V. Quantitative action of penicillin inhibitor from penicillin-resistant strains of staphylococci. Science 1945;102:221.

21 Barber M. Methicillin-resistant staphylococci. f Clin Pathol 1961;14:385.

22 Bell SM. Recommendations for the control of the spread of methicillin-resistant Staphylococcus aureus infection. Based on 18 years' experience in a group of teaching hospitals. Med $\mathcal{F}$ Aust 1982;1:472-4.

23 Kerr S, Kerr GE, Mackintosh CA, Marples RR. A survey of methicillin-resistant Staphylococcus aureus affecting pa-
tients in England and Wales. $\mathcal{F}$ Hosp Infect 1990;16:35-48.

24 Duckworth G. Revised guidelines for the control of epidemic methicillin-resistant Staphylococcus aureus. epidemic methicillin-resistan

25 Epidemic methicillin resistant Staphylococcus aureus. Communicable Disease Report 1995;5:165.

26 Schwalbe RS, Stapleton JT, Gillingan PH. Emergence of vancomycin resistance in coagulase-negative staphylococci. N Engl f Med 1987;316:927-31.

27 Kaatz GW, Seo SM, Dorman NJ, Lerner SA. Emergence of teicoplanin resistance during therapy of Staphylococcus aureus endocarditis. $\mathcal{F}$ Infect $D$ is 1990;162:103-8.
28 Noble WC, Virani Z, Gee GA. Co-transfer of vancomycin and resistant genes from Enterococcus faecalis NCTC 12201 to Staphylococcus aureus. FEMS Microbiol Lett 1992;93: 195-8.

29 Centers for Disease Control and Prevention. Nosocomial enterococci resistant to vancomycin - United States, enterococci resistant to vancomycin

30 Moellering RC. The enterococcus: a classic example of the impact of antimicrobial resistance on therapeutic options. f Antimicrob Chemother 1991;28:1-12.

31 Williamson $R$, LeBouguénec C, Gutmann L, Horaud T. One or two low affinity penicillin-binding proteins may be responsible for the range of susceptibility of Enterococcus
faecium to benzylpenicillin. f Gen Microbiol 1985; 131:1933-40.

32 Uttley AHC, Collins CH, Naidoo J, George RC. Vancomycin-resistant enterococci. Lancet 1988;i:57-8.

33 Leclercq R, Derlot E, Duval J, Courvalin P. Plasmidmediated resistance to vancomycin and teicoplanin in Enterococcus faecium. $N$ Engl f Med 1988;319:157-61.

34 Arthur M, Courvalin P. Genetics mechanisms of glycopeptide resistance in enterococci. Antimicrob Agents Chemother 1993;37:1563-71.

35 Shay DK, Maloney SA, Montecalvo M, Baneriee S, Wormser GP, Ardiuno MJ, et al. Epidemiology and mortality risk of vancomycin-resistant enterococcal bloodstream infections. $\mathcal{f}$ Infect Dis 1995;172:993-1000.

36 Jordens JZ, Bates J, Griffiths DT. Faecal carriage and nosocomial spread of vancomycin-resistant Enterococcus faecalis. $\mathcal{F}$ Antimicrob Chemother 1994;34:515-28.

37 Freiden TR, Munsiff SS, Low DE, Willey BM, Williams G, Faur Y, et al. Emergence of vancomycin-resistant enterococci in New York City. Lancet 1993;342:76-9.

38 Korten V, Murray BE. The nosocomial transmission of enterococci. Curr Opin Infect Dis 1993;6:498-505.

39 Murray BE. Editorial response: What can we do about vancomycin-resistant enterococci? Clin Infect Dis 1995; 20:1134-6.

40 Recommendations for preventing the spread of vancomycin-resistance. Recommendations of the Hospital Infection Control Practices Advisory Committee (HICPAC). MMWR 1995;44:1-13.

$41 \mathrm{McF}$ arlane J. The clinical impact of pneumococcal disease. In: Mayon-White RT, ed. The clinical impact of pneumococcal disease and strategies for its prevention. International congress and Symposium Series No 210. London: Royal Society of Medicine Press Ltd, 1995:9-17.

42 Aszkenasy OM, George RC, Begg NT. Pneumococcal bacteraemia and meningitis in England and Wales 1982 to 1992. Communicable Disease Report 1995;5:R45-50.

43 Lister PD. Multiply-resistant pneumococcus: therapeutic problems in the management of serious infections. Eur $\mathcal{F}$ Clin Microbiol Infect Dis 1995;14(Suppl 1):18-25.

44 Appelbaum PC. Antimicrobial resistance in Streptococcus pneumoniae: An overview. Clin Infect Dis 1995;15:77-83.

45 Hofman J, Cetron MS, Farley MM, Baughman WS Facklam RR, Elliott JA, et al. The prevalence of drug-resistant Streptococcus pneumoniae in Atlanta. $N$ Engl $\mathcal{F}$ Med 1995;333:481-6.

46 Geslin P, Buu-Hoi A, Fremaux A, Acar JF. Antimicrobial resistance in Streptococcus pneumoniae: An epidemiological survey in France, 1970-1990. Clin Infect Dis 1992. 15:95-8.

47 Lee HJ, Park JY, Jang SH, Kim JH, Kim EC, Choi KW, et al. High incidence of resistance to multiple antimicrobials in High incidence of resistance to multiple antimicrobials in clinical isolates of Streptococcus pneumoniae from a uni-

48 Antimicrobial resistance of pneumococi isolated from blood and cerebrospinal fluid 1993 and 1994. Communicable Disease Report 1995;5:187-8.

49 Coffey TJ, Dowson CG, Daniels M, Spratt B. Genetics and molecular biology of $\beta$-lactam-resistant pneumococci. Microbial Drug Resistance 1995;1:29-34.

50 McDougal LK, Facklam R, Reeves M, Hunter S, Swenson $\mathrm{JM}$, Hill BC, et al. Analysis of multiple antimicrobialresistant isolates of Streptococcus pneumoniae from the
United States. Antimicrob Agents Chemother 1992;36:217684.

51 Caputo GM, Appelbaum PC, Liu HH. Infections due to penicillin-resistant pneumococci. Arch Intern Med 1993; 153:1301-10.

52 Pallares R, Linares J, Vadillo M, Cabellos C, Manresa F, Viladrich PF, et al. Resistance to penicillin and cephalosporin and mortality from severe pneumococcal pneumonia in Barcelona, Spain. N Engl f Med 1995;333:47480.

53 Friedland IR, McCracken GH. Management of infections caused by antibiotic-resistant Streptococcus pneumoniae. N Engl भ Med 1994;331:377-82.

54 John CC. Treatment failure with use of a third-generation cephalosporin for penicillin-resistant pneumococcal meningitis: case report and review. Clin Infect Dis 1994;18:18893.

55 Johnson AP, Speller DCE, Patel BC. Sensitivity to cefotaxime of pneumococci isolated in the UK. $f$ Antimicrob Chemother 1995;35:443-4.

56 Taylor I, Tomlinson AJH, Davies JR. Diphtheria control in the 1960's. $\mathcal{F} R$ Soc Health 1962;82:158-64.

57 White JM, Leon S. 'COVER' (Cover of vaccination evaluated rapidly): 34. Communicable Disease Report 1995; 5:R105-6. 
58 A case of diphtheria from Pakistan. Communicable Disease Report 1994;4:173.

59 Maurice J. Belated attempts to contain Russia's diphtheria. Lancet 1995;345:715.

60 Maple PA, Efstratiou A, George RC, Andrews NJ, Sesardic D. Diphtheria immunity in UK blood donors. Lancet 1995; 345:963-5.

61 Miller E, Rush M, Morgan-Capner P, Hutchinson D, Hindle L. Immunity to diphtheria in adults in England. BMF 1994;308:598.

$62 \mathrm{Begg}$ N. WHO Manual for the management and control of diphtheria in the European Region.1994;ICP/EPI 038.

63 Department of Health. Diphtheria immunisation and treatment. CMO's Update 1995;5:1.

64 Efstratiou A, George RC, Begg NT. Non-toxigenic Corynebacterium diphtheriae var gravis in England. Lancet 1993; 341:1592-3.
65 Wilson APR. The return of Corynebacterium diphtheriae: the rise of non-toxigenic strains. $\mathcal{F}$ Hosp Infect 1995; 30(Suppl A):306-12.

66 Gruner E, Opravil M, Altvegg $M$, von Graevenitz A. Nontoxigenic Corynebacterium diphtheriae isolated from intravenous drug users. Clin Infect Dis 1994;18:94-6.

67 Zuber PLF, Gruner E, Altwegg M, von Graevenitz A. Invasive infection with non-toxigenic Corynebacterium diphtheriae among drug users. Lancet 1992;339:1359.

68 Tiley SM, Kociuba KR, Heron LG, Munro R. Infective endocarditis due to non-toxigenic Corynebacterium diphtheriae: Report of seven cases and review. Clin Infect Dis 1993;16:271-5.

69 Freeman VJ. Studies on the virulence of bacteriophageinfected strains of Corynebacterium diphtheriae. $\mathcal{F}$ Bacteriol 1951;61:675-88. 